\title{
The effect of progesterone and pregnenolone on diabetes status in Chinese rural population: a dose-response analysis from Henan Rural Cohort
}

\author{
Jingjing Jiang ${ }^{1,2}$, Xue Liu' ${ }^{1}$ Xiaotian Liu' ${ }^{1}$, Zhongyan Tian'1, Haiqing Zhang', Xinling Qian', Zhicheng Luo', \\ Dandan Wei', Shuna Jin ${ }^{3}$, Chongjian Wang' ${ }^{1}$ and Zhenxing Mao' ${ }^{1}$
}

${ }^{1}$ Department of Epidemiology and Biostatistics, College of Public Health, Zhengzhou University, Zhengzhou, Henan, People's Republic of China, ${ }^{2}$ Medical Insurance Office, Liuzhou General Hospital, Liuzhou, Guangxi, People's Republic of China, and ${ }^{3}$ Key Laboratory of Environment and Health, Ministry of Education \& Ministry of Environmental Protection, and State Key Laboratory of Environmental Health, School of Public Health, Tongji Medical College, Huazhong University of Science and Technology, Wuhan, Hubei, People's Republic of China

Correspondence should be addressed to Z Mao Email maozhr@gmail.com

\begin{abstract}
Objective: Previous studies have uncovered a progestin-only contraceptive association with an increased risk of diabetes, but limited studies have explored the relationship of endogenous progesterone and pregnenolone levels with diabetes status. A case-control study was conducted in Henan Rural Cohort (register number: ChiCTROOC-15006699) to evaluate the dose-response independent and interactive relationship of progesterone and pregnenolone levels with prediabetes and type 2 diabetes mellitus (T2DM) in Chinese rural population.

Design: A case-control study.

Methods: A total of 798 T2DM patients, 779 prediabetes patients, and 782 individuals with normal fasting plasma glucose were included in this study. Serum progesterone and pregnenolone were detected by liquid chromatographytandem mass spectrometry. Logistic regression and restricted cubic splines were used to assess the independent effects of progesterone and pregnenolone on prediabetes and T2DM. Interactive plots were employed to examine the interaction effects of progesterone and pregnenolone.

Results: Progesterone in the fourth versus first quartile was positively associated with prediabetes (odds ratio (OR) (95\% Cl): 2.66 (1.99-3.55)) and T2DM (OR (95\% CI): 6.41 (4.57-8.98)), whereas pregnenolone in the fourth versus first quartile was inversely related to prediabetes (OR (95\% CI): $0.23(0.16-0.33)$ ) and T2DM (OR (95\% Cl): $0.44(0.31-0.62)$ ). Additionally, the nonlinear dose-response associations between progesterone and pregnenolone with prediabetes and T2DM were found. Interactive effects of progesterone and pregnenolone on prediabetes and T2DM were observed, and these significant associations remained in gender-stratified analysis.

Conclusions: Prediabetes and T2DM were positively linked to serum concentration of progesterone and negatively related to pregnenolone in a dose-response manner in Chinese rural population.
\end{abstract}




\section{Introduction}

Diabetes mellitus is one of the most common causes of mortality and disability $(1,2,3)$, affecting more than 425 million individuals worldwide (http://diabetesatlas. org/key-messages.html, accessed 11 September 2018) (4). Moreover, prediabetes, characterized by elevated blood glucose levels and intermediate status between normal and diabetic status, is associated with an increased risk of diabetes and cardiovascular disease (5). Most importantly, individuals with prediabetes can be prevented from developing diabetes, and can even be reverted to normal. The rates of diabetes and prediabetes were estimated to be 10.9 and $35.7 \%$ in China in 2013 and have increased alarmingly (6). Notably, the prevalence of diabetes has escalated more rapidly in rural population than in urban population in China (7). Type 2 diabetes mellitus (T2DM) accounts for approximately $90 \%$ of the individuals with diabetes $(1,2)$. Hence, it is urgent and crucial to identify the factors that influence the development of prediabetes and T2DM.

Previous researches have indicated that endocrine hormones are associated with the development of T2DM $(8,9)$. Progesterone, the principal progestogen, is associated with obesity, abnormal blood lipid levels, and increased blood pressure, all of which are related to T2DM $(10,11,12)$. Several studies have shown that high concentration of progesterone is related to abnormal glucose metabolism, including a high level of plasma glucose, increased insulin resistance, elevated insulin release and apoptosis of pancreatic $\beta$ cells $(13,14,15,16)$. Pregnenolone, the immediate precursor of progesterone and also a progestogen, is also associated with the blood level of insulin (17). Interestingly, gestational diabetes generally presents with high levels of progesterone in the second trimester (14). Moreover, several studies have demonstrated that progestogen-only contraceptives may play a crucial role in the development of diabetes $(18,19,20)$. Additionally, a finding from a recent metaanalysis indicated that women taking supplemental 17-OH progesterone caproate were related to an elevated risk of gestational diabetes mellitus (21). However, there is little information on the relationship of endogenous progestogen with prediabetes or diabetes. Up to date, only one epidemiological study, which focused on elderly opposite-sex twins in a Swedish population, assessed the association of serum concentration of progesterone with the risk of diabetes, but no significant association was found (22). To the best of our knowledge, no populationbased study has been carried out to explore the relationship of endogenous progesterone and pregnenolone levels with the risk of prediabetes.

Therefore, this study was conducted to explore the dose-response independent and interactive associations of endogenous (serum) progesterone and pregnenolone with the risk of prediabetes and T2DM in Chinese rural population.

\section{Subjects and methods}

\section{Study design and participants}

Data for the current study were extracted from the baseline data (from July 2015 to September 2017) of the Henan Rural Cohort (register number: ChiCTR-OOC-15006699), an ongoing prospective study designed to explore the potential risk factors of common chronic diseases in Chinese rural population (23). On the basis of American Diabetes Association (ADA) diagnostic criteria (2009) (5) and the WHO criteria (1999) (24), after excluding type 1 diabetes mellitus, and diabetes caused by other reasons, T2DM was defined as subjects with diagnosis of T2DM by physician and taking antidiabetic drugs or insulin during the past fortnight, or fasting plasma glucose (FPG) $\geq 7.0 \mathrm{mmol} / \mathrm{L}$, or glycosylated hemoglobin (HbA1c) $\geq 6.5 \%$; prediabetes was determined as individuals without T2DM and $\mathrm{FPG} \geq 6.1 \mathrm{mmol} / \mathrm{L}$ or $\mathrm{HbA} 1 \mathrm{c} \geq 5.7 \%$.

A total of 2359 participants were included in this study: 782 individuals with T2DM, 779 prediabetes patients, and 798 control individuals. Prediabetes patients and control individuals were sampled randomly and frequency matched to participants with T2DM according to age (3-year groups) and gender. Men and postmenopausal women aged from 18 to 79 years were included in this study. Premenopausal women were not included due to the significant effect of the menstrual cycle on progestogen blood levels. Individuals with missing or insufficient serum samples to detect progestogen were ruled out. Participants with hormone replacement therapy (HRT) were also excluded because of the potential impact of HRT on progestogen levels.

\section{Ethics approval and consent to participate}

Ethics approval was obtained from the 'Zhengzhou University Life Science Ethics Committee', and written informed consent was obtained from all participants. Ethic approval code: (2015) MEC (S128).

The protocol of this study was approved by Zhengzhou University Life Science Ethics Committee and conducted 
according to the guidelines of the Declaration of Helsinki. All participants provided the informed written consents after they were told the purpose and nature of all procedures used.

\section{Covariates}

Information on sociodemographic characteristics, including age, gender, marital status (married/ cohabitating and unmarried/divorced/widowed), education level (below than primary school, primary school, middle school or above), average monthly income (<500, 500 , 1000 RMB), family history of diabetes, usage of HRT, behavioral habits including smoking habit (current smoker, never or former), alcohol drinking habit (current drinker, never or former), and physical activity, were collected by well-trained interviewers with a structured questionnaire. Women were further requested to provide information on their menopausal status. Based on International Physical Activity Questionnaire (IPAQ) (25), physical activity was divided into three groups: low, mediate and high level. Parents or siblings of participants with a history of diabetes were considered to be with a family history of diabetes. Height and body weight were measured by trained investigators. BMI was calculated as weight $(\mathrm{kg})$ divided by height $(\mathrm{m})$ squared.

\section{Laboratory measurements}

After an overnight fasting, venous blood samples of participants were collected. Serum was separated after centrifugation at $1409 \boldsymbol{g}$ in $4^{\circ} \mathrm{C}$ for $10 \mathrm{~min}$ and stored at $-80^{\circ} \mathrm{C}$. An automatic biochemical analyzer (Roche Cobas C501) was used to measure the concentration of FPG, triglycerides (TG), total cholesterol (TC), highdensity lipoprotein cholesterol (HDL-C), and low-density lipoprotein cholesterol (LDL-C). HbA1c was measured using HPLC method (Bio-Rad VARIANT II). The intraand inter-assay coefficients of variation (CVs) were less than 3\% for above-mentioned laboratory measurements (Supplementary Table 1, see section on supplementary data given at the end of this article). The updated homeostasis model (HOMA2) was used to estimate insulin resistance and $\beta$-cell function with the corresponding indexes, namely HOMA2-IR and HOMA2- $\beta$ (https://www. dtu.ox.ac.uk/homacalculator/) (26).

The serum level of progesterone and pregnenolone was determined by liquid chromatography-tandem mass spectrometry method (Waters e2695, Waters XEVO TQ-S system, Waters), blinded to T2DM, prediabetes, or control status $(27,28)$; the intra- and inter-day CVs were 1.12 and $1.48 \%$ for progesterone, and the corresponding values for pregnenolone were 9.78 and $11.68 \%$ respectively. The limits of quantification were $0.05 \mathrm{ng} / \mathrm{mL}$ and $0.4 \mathrm{ng} / \mathrm{mL}$ for progesterone and pregnenolone respectively. Moreover, the detection limits of progesterone and pregnenolone were $0.02 \mathrm{ng} / \mathrm{mL}$ and $0.2 \mathrm{ng} / \mathrm{mL}$. A detailed description of the detection methods is presented in Supplementary Tables 2 and 3. According to gender-specific quartiles of the control group, progesterone and pregnenolone were classified into four groups with the lowest quartile groups as the reference groups: Quartile 1 (Q1, the first/lowest quartile), Quartile 2 (Q2, the second quartile), Quartile 3 (Q3, the third quartile), and Quartile 4 (Q4, the fourth/ highest quartile).

\section{Statistical analysis}

Because of the skewed distributions of all continuous covariates (Kolmogorov-Smirnov test: $P<0.05$ for all continuous covariates), differences between case and control individuals in continuous variables were compared using Mann-Whitney $U$ tests and expressed as medians (interquartile range (IQR)), whereas differences in categorical variables were compared using $\chi^{2}$ tests and presented as numbers (percentages).

Logistic regression was employed to estimate odds ratios (ORs) with 95\% CIs for prediabetes and T2DM by quartiles of progesterone and pregnenolone. Covariates in adjusted model included age, gender, marital status, education level, average monthly income, smoking habit, alcohol drinking habit, physical activity, BMI, family history of diabetes, TC, TG, HDL-C, and LDL-C. Furthermore, trend tests were used to assess the doseresponse relationships by including quartiles as continuous variables. Restricted cubic splines were conducted to estimate the dose-response associations of continuous progesterone and pregnenolone with the risk of prediabetes and T2DM, with three knots placed at 30th, 60th, and 90th percentiles; $0.5 \mathrm{ng} / \mathrm{mL}$ and $1.0 \mathrm{ng} / \mathrm{mL}$ were set as the reference values for progesterone and pregnenolone based on their distribution. Linear regression was performed to further explore the relationship of progesterone and pregnenolone levels with glucose homeostasis markers, including FPG, HbA1c, fasting insulin, HOMA2-IR, and HOMA2- $\beta$. In linear regression model, individuals taking hypoglycemic drugs during past 14 days were ruled out, and progesterone, pregnenolone, and glucose homeostasis markers were natural log-transformed (ln-) in order to improve normalization. To further examine the 
independent association of progestogen with prediabetes and T2DM, HOMA2-IR, HOMA2- $\beta$, and fasting insulin were separately added to adjusted logistic regression model. Additionally, the interaction effects of progesterone and pregnenolone on prediabetes and T2DM were tested by generalized linear model and visualized in Contour plot (29). Gender-stratified analysis was employed throughout the research. The interaction of progestogen and gender was also tested.

Analyses were performed using SAS 9.1 (SAS Institute Inc.) and $\mathrm{R}$ Language software (version 3.4.4). $P<0.05$ (two-tailed) was defined as statistical significance.

\section{Results}

\section{Characteristics of participants}

A total of 2359 individuals with a median age (IQR) of 62.00 (12.00) were included in this study. Characteristics of participants according to diabetes status by gender are shown in Table 1. Individuals with prediabetes and T2DM tended to have higher BMI, higher FPG, higher insulin, higher HbA1C, higher TC, and higher TG compared with controls. Diabetic individuals were more likely to have a family history of diabetes, and participants with prediabetes were more likely to have a higher LDL-C. Compared with the control group, the concentration of progesterone was higher in the prediabetes and T2DM groups, whereas the concentration of pregnenolone in the prediabetes and T2DM group was lower than that in the control group. A similar distribution was seen in men and postmenopausal women.

\section{Effects of progesterone and pregnenolone on prediabetes and T2DM}

Estimated ORs for prediabetes and T2DM are presented in Table 2. A graded increment in ORs of prediabetes across progesterone quartiles was observed $(P$ trend <0.001; Q2: OR=1.30, 95\% CI: 0.95-1.77; Q3: OR=1.73, 95\% CI: 1.28-2.33; Q4: OR=2.84, 95\% CI: 2.15-3.76), and the tendency was not significantly attenuated after further adjustment for potential confounders. Similarly, a positively dose-response relationship was found between progesterone levels and T2DM. The significantly positive associations between progesterone with prediabetes and T2DM persisted after stratification by sex.

However, pregnenolone was inversely associated with prediabetes (OR (95\% CI): Q3 vs Q1: 0.34 (0.25-0.45);
Q4 vs Q1: $0.24(0.17-0.33))$ and T2DM (OR (95\% CI): Q3 vs Q1: 0.44 (0.33-0.59); Q4 vs Q1:0.39 (0.29-0.53)); these relationships were slightly altered but still remained even after adjusting for potential confounders. Dose-response relationships were found between pregnenolone levels with the risk of prediabetes and T2DM before and after controlling for potential confounders $(P<0.001)$. The negative relationship between pregnenolone with the risk of prediabetes and T2DM was also observed in genderstratified analysis.

As shown in Fig. 1, ORs of prediabetes and T2DM increased gradually with an incremental increase in the serum concentration of progesterone in all participants, in men, and in postmenopausal women (all $P$ for overall association $<0.001$ and all $P$ for nonlinearity $<0.001$ ), indicating a significant nonlinear dose-response relationship of progesterone with prediabetes and T2DM. In contrast, ORs of prediabetes were decreased with the increment of pregnenolone in all participants, and the relationship remained even after gender stratification (all $P$ for overall association $<0.001$, all $P$ for nonlinearity $<0.001)$. A similar association was obtained in T2DM in the total population $(P$ for overall association $=0.050, P$ for nonlinearity $=0.015$ ), although the trend was weaker. Gender-stratified analysis showed that the relationship was enhanced in men $(P$ for overall association $=0.018$, $P$ for nonlinearity $=0.005)$, but disappeared in postmenopausal women $(P$ for overall association $=0.639$, $P$ for nonlinearity $=0.360$ ).

\section{Effects of progesterone and pregnenolone on glucose homeostasis markers}

Linear regression results of the relationship between ln-progesterone and ln-pregnenolone with glucose homeostasis markers are displayed in Fig. 2 and Supplementary Table 4. A 100\% increase in ln-progesterone was related to a $0.052 \mathrm{mmol} / \mathrm{L}$ (95\% CI: 0.037 0.066) higher ln-FPG, 3.6\% (95\% CI: 2.6 4.5\%) higher ln-HbA1c, and 9.2\% (95\% CI: 6.3 12.1\%) lower ln-HOMA2- $\beta$ after adjusting for potential confounders. Similarly, compared with the lowest quartile of ln-progesterone, the highest quartile of $\ln$-progesterone was associated with 0.110 mmol/L(95\% CI: 0.082 0.137) higher ln-FPG, and 7.2\% (95\% CI: 5.3 9.0\%) higher ln-HbA1c, whereas a $20.9 \%$ (95\% CI: 15.1 26.6\%) lower In-HOMA2- $\beta$. Genderstratified analysis indicated that the relationship was slightly stronger in postmenopausal women and slightly weaker in men. 


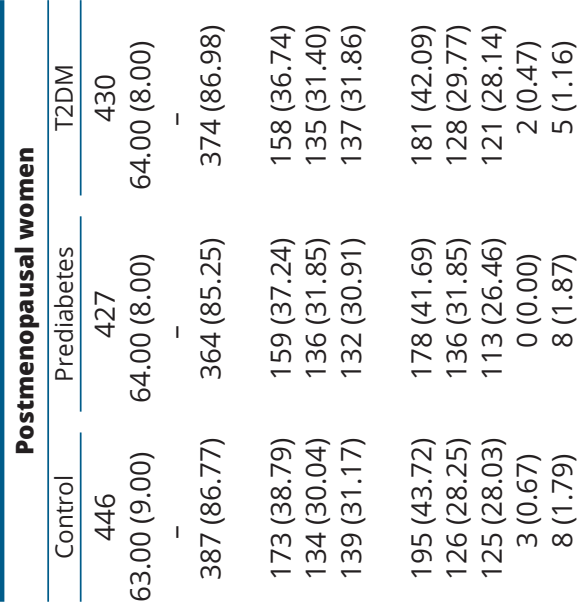

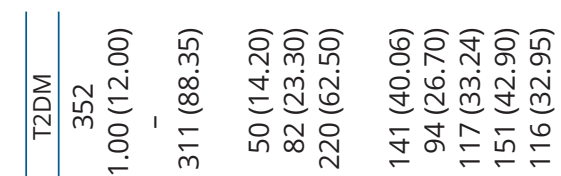

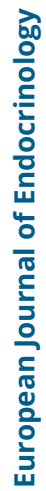

ำลำสำ

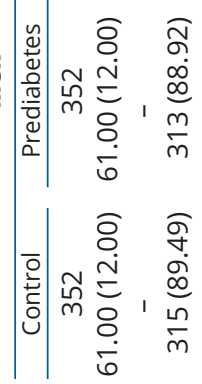

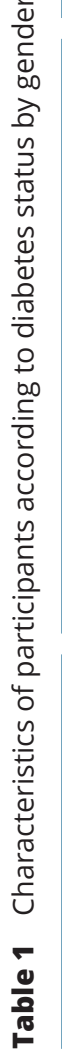

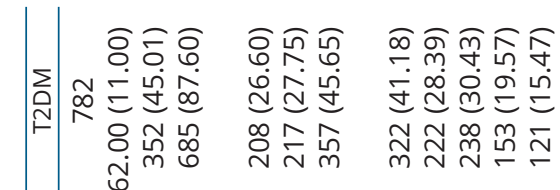

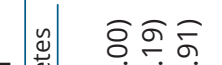

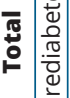

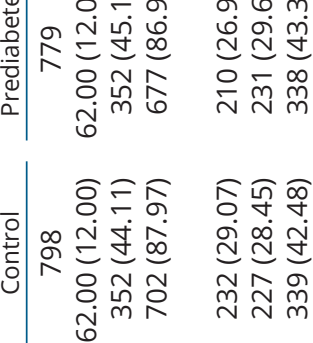

ริดส

ऽ

的永卷

종

눙

ํํㅇㅇㅠ mं

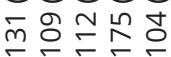

môำ 过它过守过 능 $8 \infty \infty$

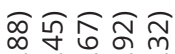

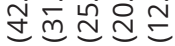

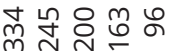
过守主过

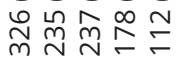

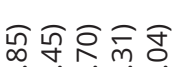

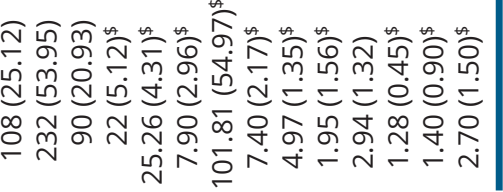

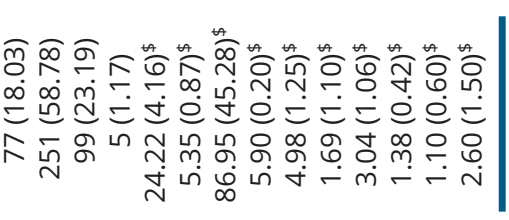

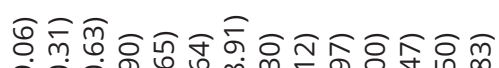

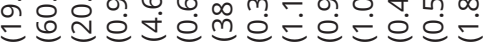

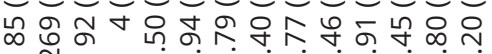

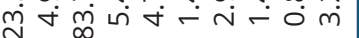

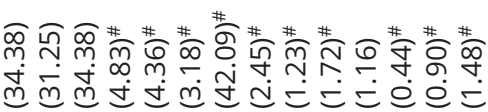

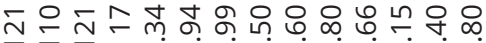

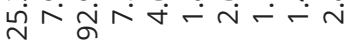

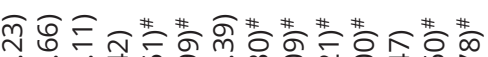

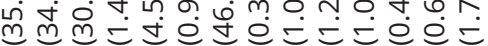
ป 쥼

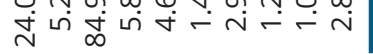

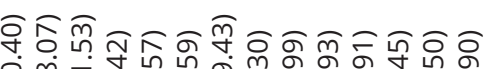

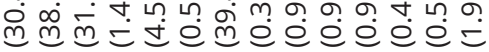

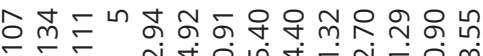
ปัنठ் ம்

范

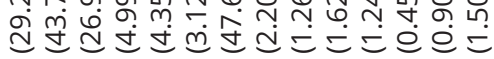

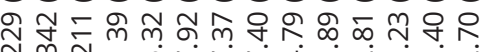

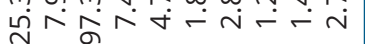

o

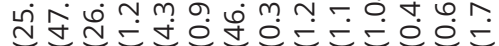
휬

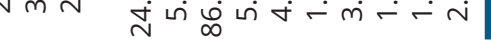

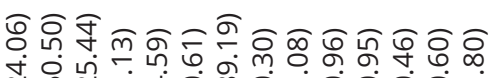

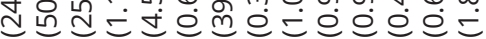
๙

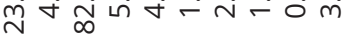

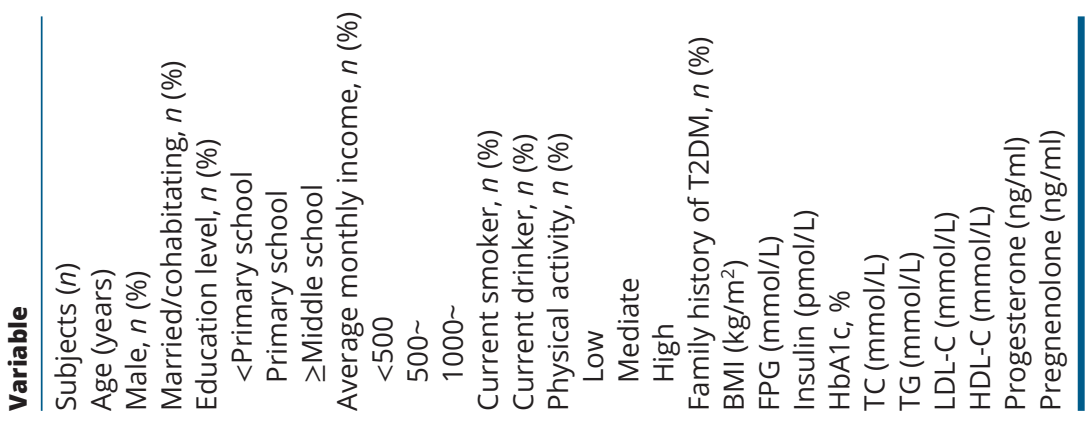

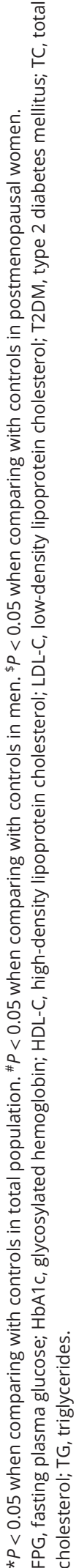


Table 2 Odds ratios (95\% confidence intervals) of prediabetes/T2DM.

\begin{tabular}{|c|c|c|c|c|}
\hline \multirow[b]{2}{*}{ Variable } & \multicolumn{2}{|c|}{ Prediabetes } & \multicolumn{2}{|c|}{ T2DM } \\
\hline & Unadjusted ORs (95\% Cls) & Adjusted ORs $(95 \% \mathrm{Cls})^{\dagger}$ & Unadjusted ORs (95\% Cls) & Adjusted ORs $(95 \% \mathrm{Cls})$ \\
\hline \multicolumn{5}{|l|}{ Progesterone } \\
\hline \multicolumn{5}{|l|}{ Total } \\
\hline Q1 & 1.00 & 1.00 & 1.00 & 1.00 \\
\hline Q2 & $1.30(0.95-1.77)$ & $1.26(0.92-1.74)$ & $0.93(0.63-1.39)$ & $0.88(0.57-1.35)$ \\
\hline Q3 & $1.73(1.28-2.33)^{\star \star *}$ & $1.58(1.16-2.15)^{* *}$ & $2.21(1.56-3.11)^{* * *}$ & $1.95(1.34-2.84)^{\star * *}$ \\
\hline Q4 & $2.84(2.15-3.76)^{* * *}$ & $2.66(1.99-3.55)^{* * *}$ & $7.34(5.39-10.01)^{\star \star \star}$ & $6.41(4.57-8.98)^{* \star *}$ \\
\hline$P$ for trend & $<0.001$ & $<0.001$ & $<0.001$ & $<0.001$ \\
\hline \multicolumn{5}{|l|}{ Men } \\
\hline Q1 & 1.00 & 1.00 & 1.00 & 1.00 \\
\hline Q2 & $1.09(0.68-1.75)$ & $1.12(0.69-1.84)$ & $0.92(0.52-1.63)$ & $0.87(0.46-1.64)$ \\
\hline Q3 & $1.51(0.96-2.36)$ & $1.46(0.91-2.35)$ & $1.86(1.11-3.10)^{*}$ & $1.84(1.03-3.27)^{\star}$ \\
\hline Q4 & $2.16(1.43-3.28)^{* * *}$ & $2.13(1.37-3.31)^{* *}$ & $5.54(3.51-8.75)^{* * *}$ & $4.47(2.67-7.47)^{\star * *}$ \\
\hline$P$ for trend & $<0.001$ & $<0.001$ & $<0.001$ & $<0.001$ \\
\hline \multicolumn{5}{|c|}{ Postmenopausal women } \\
\hline Q1 & 1.00 & 1.00 & 1.00 & 1.00 \\
\hline Q2 & $1.48(0.97-2.26)$ & $1.39(0.90-2.14)$ & $0.91(0.52-1.60)$ & $0.81(0.44-1.48)$ \\
\hline Q3 & $1.92(1.28-2.87)^{\star \star}$ & $1.70(1.12-2.57)^{*}$ & $2.52(1.58-4.02)^{\star \star *}$ & $2.00(1.21-3.32)^{\star *}$ \\
\hline Q4 & $3.54(2.42-5.17)^{* * *}$ & $3.21(2.17-4.75)^{\star * *}$ & $9.21(6.03-14.06)^{\star \star *}$ & $8.41(5.34-13.24)^{\star * *}$ \\
\hline$P$ for trend & $<0.001$ & $<0.001$ & $<0.001$ & $<0.001$ \\
\hline \multicolumn{5}{|l|}{ Pregnenolone } \\
\hline \multicolumn{5}{|l|}{ Total } \\
\hline Q1 & 1.00 & 1.00 & 1.00 & 1.00 \\
\hline Q2 & $1.01(0.79-1.30)$ & $1.02(0.79-1.32)$ & $1.20(0.93-1.55)$ & $1.16(0.88-1.54)$ \\
\hline Q3 & $0.34(0.25-0.45)^{\star * *}$ & $0.33(0.25-0.45)^{\star * *}$ & $0.44(0.33-0.59)^{* * *}$ & $0.48(0.35-0.66)^{\star * *}$ \\
\hline Q4 & $0.24(0.17-0.33)^{\star * *}$ & $0.23(0.16-0.33)^{\star * *}$ & $0.39(0.29-0.53)^{\star * *}$ & $0.44(0.31-0.62)^{* * *}$ \\
\hline$P$ for trend & $<0.001$ & $<0.001$ & $<0.001$ & $<0.001$ \\
\hline \multicolumn{5}{|l|}{ Men } \\
\hline Q1 & 1.00 & 1.00 & 1.00 & 1.00 \\
\hline Q2 & $0.98(0.67-1.44)$ & $1.01(0.68-1.50)$ & $1.02(0.70-1.49)$ & $0.89(0.58-1.37)$ \\
\hline Q3 & $0.36(0.23-0.56)^{\star \star *}$ & $0.36(0.23-0.58)^{* * *}$ & $0.33(0.21-0.52)^{\star * *}$ & $0.38(0.23-0.63)^{* \star *}$ \\
\hline Q4 & $0.22(0.13-0.35)^{\star * *}$ & $0.21(0.13-0.36)^{* * *}$ & $0.28(0.18-0.45)^{* * *}$ & $0.32(0.19-0.54)^{* * *}$ \\
\hline$P$ for trend & $<0.001$ & $<0.001$ & $<0.001$ & $<0.001$ \\
\hline \multicolumn{5}{|c|}{ Pregnenolone } \\
\hline \multicolumn{5}{|c|}{ Postmenopausal women } \\
\hline Q1 & 1.00 & 1.00 & 1.00 & 1.00 \\
\hline Q2 & $1.03(0.74-1.45)$ & $1.04(0.73-1.47)$ & $1.39(0.98-1.97)$ & $1.41(0.96-2.05)$ \\
\hline Q3 & $0.32(0.21-0.48)^{\star \star *}$ & $0.30(0.20-0.46)^{\star * *}$ & $0.55(0.37-0.81)^{* *}$ & $0.57(0.37-0.87)^{\star *}$ \\
\hline Q4 & $0.25(0.16-0.39)^{* * *}$ & $0.25(0.16-0.39)^{* * *}$ & $0.52(0.34-0.78)^{* *}$ & $0.56(0.36-0.89)^{*}$ \\
\hline$P$ for trend & $<0.001$ & $<0.001$ & $<0.001$ & $<0.001$ \\
\hline
\end{tabular}

${ }^{\dagger}$ Adjusted for age, gender (only in total population), marital status, education level, average monthly income, smoking habit (in total population and in men), alcohol drinking habit (in total population and in men), physical activity, BMI, and family history of T2DM, total cholesterol, triglycerides, low-density lipoprotein cholesterol, HDL-C. *Significant $P<0.05$, ** Significant $P<0.01, * * \star$ Significant $P<0.001$.

$95 \% \mathrm{Cl}, 95 \% \mathrm{Cl}$; OR, odds ratio; Q1, the first/ lowest quartile; Q2, the second quartile; Q3, the third quartile; Q4, the fourth/highest quartile; T2DM, type 2 diabetes mellitus.

Ln-pregnenolone in the highest quartile vs the lowest quartile was associated with a $4.3 \%$ (95\% CI: 2.3 6.4\%) lower $\ln -\mathrm{HbA} 1 \mathrm{c}$, and a similar association was observed in men and postmenopausal women. Whereas the highest quartile of pregnenolone was associated with a $6.9 \%$ (95\% CI: 0.4 13.5\%) higher In-HOMA2- $\beta$ compared to the lowest quartile of pregnenolone. However, this relationship vanished after gender stratification.

\section{The modified effects of HOMA2-IR, HOMA2- $\beta$, and fasting insulin}

Logistic regression showed that the relationship of progestogen to prediabetes and T2DM was moderately attenuated but still remained after further adjustment for HOMA2-IR, HOMA2- $\beta$, and fasting insulin (Supplementary Table 5). 

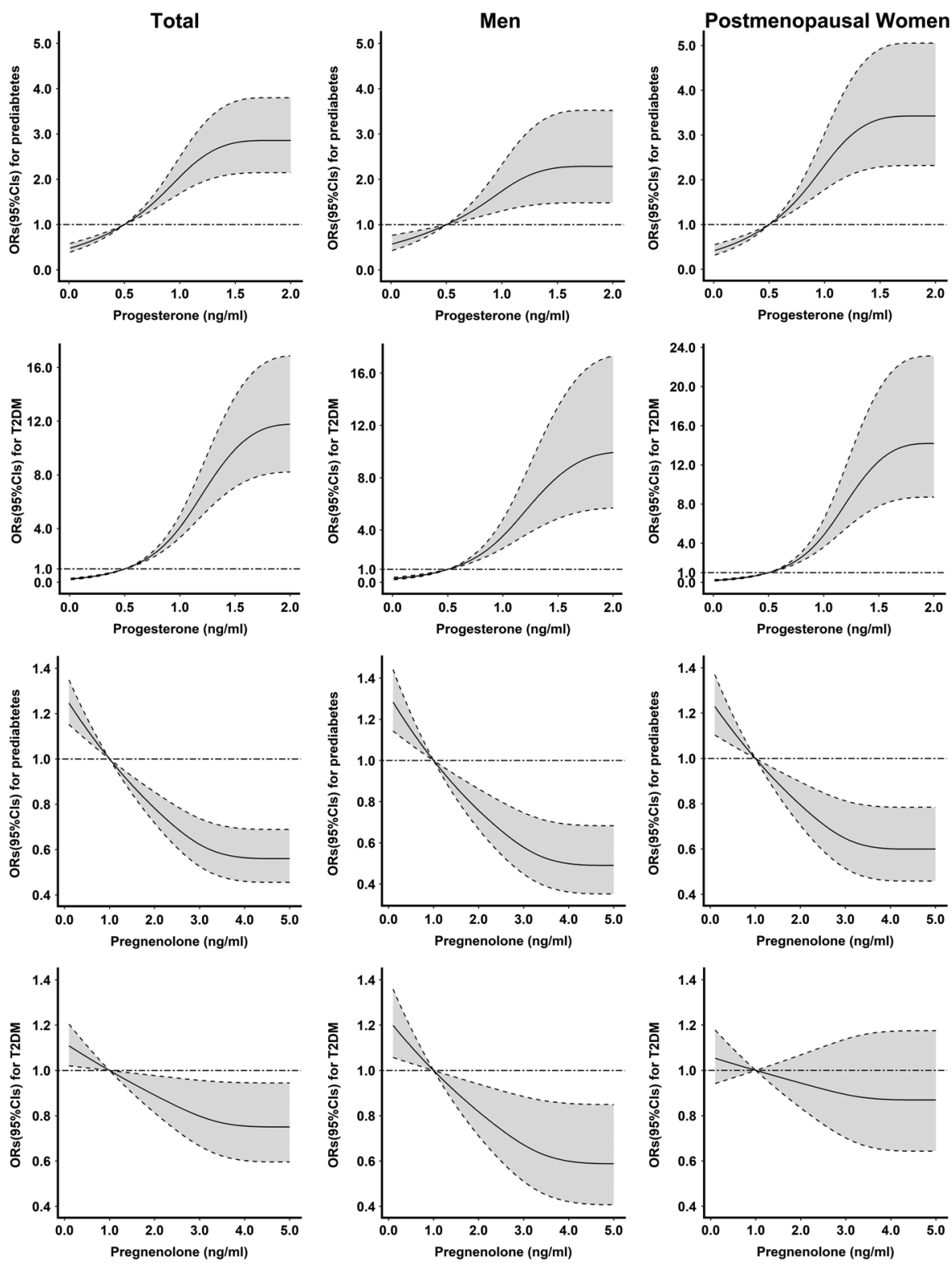

\section{Figure 1}

The dose-response associations between progesterone and pregnenolone with prediabetes and T2DM estimated by restricted cubic splines. Three knots were set at the 30th, 60th, and 90th percentiles with the reference value at $0.5 \mathrm{ng} / \mathrm{mL}$ for progesterone and $1 \mathrm{ng} / \mathrm{mL}$ for pregnenolone according to the distribution. Adjusted for age, gender (only in total population), marital status, education level, average monthly income, smoking habit (in total population and in men), alcohol drinking habit (in total population and in men), physical activity, body mass index, and family history of T2DM, total cholesterol, triglycerides, low-density lipoprotein cholesterol, high-density lipoprotein cholesterol; T2DM, type 2 diabetes mellitus; OR, odds ratio; 95\% CI, $95 \%$ confidence interval.

\section{Interaction effects}

The interaction effects of progesterone and pregnenolone on prediabetes and T2DM are shown in Fig. 3. Individuals with the highest progesterone and lowest pregnenolone were at highest risk of prediabetes; those with the lowest progesterone and highest pregnenolone were at lowest risk of prediabetes ( $P$ for interaction $<0.001$ ). Furthermore, with the increase of pregnenolone, the estimated effect value of progesterone on prediabetes was obviously 


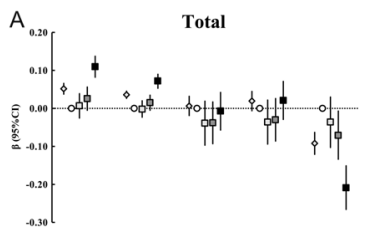

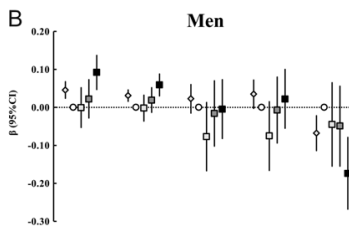

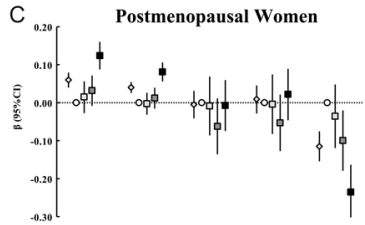

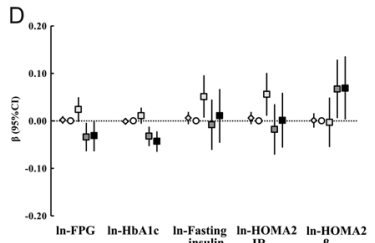

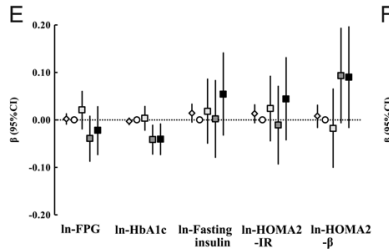<smiles>FC(F)(F)C1CC2C3CCC(C3)CC21</smiles>

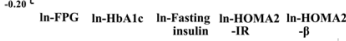

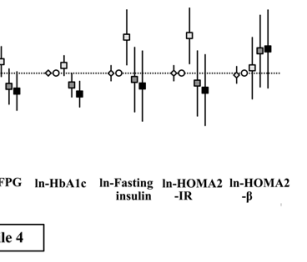

\section{Figure 2}

Association of progesterone $(A, B, C)$ and pregnenolone $(D, E, F)$ with glucose homeostasis markers. Adjusted for age, gender (only in total population), marital status, education level, average monthly income, smoking habit (in total population and in men), alcohol drinking habit (in total population and in men), physical activity, body mass index, and family history of T2DM, total cholesterol, triglycerides, low-density lipoprotein cholesterol, high-density lipoprotein cholesterol. FPG, HbA1c, fasting insulin, HOMA2-IR, HOMA2- $\beta$, progesterone, and pregnenolone were natural logtransformed (In) in order to improve normalization. The updated homeostasis model (HOMA2) was used to estimate insulin resistance and $\beta$-cell function with the corresponding indexes, namely HOMA2-IR and HOMA2- $\beta$. 95\% Cl, 95\% confidence interval; FPG, fasting plasma glucose; HbA1c, glycosylated hemoglobin.

\section{Figure 3}

The interaction effects of progesterone and pregnenolone on prediabetes and T2DM. Contour plots estimated by generalized linear model showed the linear predictor of the hazard function. The linear predictors of the hazard function for different concentrations of progesterone and pregnenolone were exhibited in different colors, ranging from blue (low risk) to pink (high risk). Linear predictors were adjusted for age, gender (only in total population), marital status, education level, average monthly income, smoking habit (in total population and in men), alcohol drinking habit (in total population and in men), physical activity, body mass index, and family history of T2DM, total cholesterol, triglycerides, low-density lipoprotein cholesterol, high-density lipoprotein cholesterol. T2DM, type 2 diabetes mellitus. 
decreased, but the statistical significance remained. Gender-stratified analysis showed that the relationship was similar in postmenopausal women $(P$ for interaction $<0.001$ ), whereas the effect of progesterone on prediabetes was diminished and eventually disappeared in men with an increase of pregnenolone ( $P$ for interaction $=0.004)$.

The highest risk of T2DM was observed with the highest progesterone and lowest pregnenolone levels, whereas the lowest risk of T2DM was found in lowest progesterone and highest pregnenolone $(P$ for interaction $<0.001)$. Additionally, with an increment of pregnenolone the impact of progesterone on T2DM was slightly diminished but still existed. A similar association was seen after stratification according to gender ( $P$ for interaction $<0.001$ in men and in postmenopausal women).

Interactions between progestogen and gender were not found in this study (all $P>0.05$ ).

\section{Discussion}

To the best of our knowledge, this research is the first study to explore the differential relationships of endogenous progesterone and pregnenolone to diabetes status in Chinese rural population. The serum level of progesterone was positively associated with prediabetes and T2DM in a nonlinear dose-response manner, and also was related to higher FPG, higher HbA1c, as well as lower HOMA2- $\beta$. In contrast, the serum level of pregnenolone was negatively associated with prediabetes and T2DM in a nonlinear dose-response fashion and was linked to lower HbA1c. Furthermore, significant interactions between progesterone and pregnenolone on prediabetes and T2DM were observed in this study: with each increment of pregnenolone, the positive effect of progesterone on prediabetes and T2DM was diminished.

Previous research conducted in 425 elderly Swedish individuals indicated that the association between progesterone and diabetes was not significant (22), which was contrary to our findings. The prior research targeted elderly Swedish opposite-sex twins whose age ranged from 71 to 80 years. Different age range, varied ethnicity, as well as diverse economic status may explain the different conclusions. Further, the limited sample size of the Swedish study may result in a limited statistical power to detect an association. Several studies showed that the use of a progestogen-only contraceptive was associated with increased insulin resistance, decreased insulin sensitivity, and elevated risk of diabetes (18, $19,20,30,31,32,33,34)$, which partly supported our findings, as progesterone was the main progestogen with biological activity. However, prior research indicated that synthetic progestogen was different from natural progesterone: synthetic progestogen may contribute to a higher prevalence of abnormal glucose metabolism and gestational diabetes mellitus, while natural progesterone did not $(35,36,37)$. However, the findings of current study showed that serum progesterone was associated with higher FPG, higher HbA1c, and decreased betacell function, along with higher risk of prediabetes and T2DM in Chinese rural population. The difference in the results may due to the different ethnicities and disease outcomes. The current study was focused on men and postmenopausal women, while other studies aimed at pregnant women, which may result in contradictory results. Thus, a study conducted in the general population is needed to evaluate the effect of progestogen on T2DM. Additionally, an experimental research with rats reported that the effect of a high dose of glibenclamidepregnenolone on anti-hyperglycemic activity was better than glibenclamide or glibenclamide-OH, which was in consistence with the present study to some degree (38). The results of gender stratification analysis were similar to the findings in all participants, which partly may be due to the similar source of progestogen production (adrenal) in postmenopausal women and men $(39,40)$.

Individuals with prediabetes and T2DM in this study tended to be obese and with higher serum levels of progesterone. Similar to our study, a previous study reported that an increase in progesterone was observed in $\mathrm{Db} / \mathrm{db}$ mice with obesity and high blood glucose (41). Moreover, a study conducted in a Chinese population reported that individuals with prediabetes and T2DM tended to have a higher concentration of TC and TG, but lower concentration of HDL-C than individuals with normal blood glucose (42), which was in accordance with our findings.

The mechanism by which progestogen is associated with diabetes status has not been elucidated, but there are several potential explanations. Progesterone plays an important role in some physiological processes of diabetes. It contributes to the decrease of insulin release $(16,43,44)$ and insulin-secreting cell proliferation (13). An animal experiment conducted by Picard et al. showed that elevated progesterone leads to diabetes in female $D b / d b$ mice, whereas the blood glucose level was significantly improved after the administration of a progesterone receptor antagonist (16). The further experiment showed increased insulin release, improved glucose tolerance, and lager pancreatic islets in progesterone receptor-knockout 
mice. These findings suggested that progesterone had an impact on the proliferation of islet $\beta$-cells and insulin release, which may eventually lead to diabetes. Evidence from Nunes et al. (13) suggested that progesterone induced apoptotic death of insulin-producing cells by acting through a mechanism related to the generation of reactive oxygen species and oxidative stress. The findings of this study also demonstrated that higher progesterone was related to higher FPG and impaired beta-cell function, which was consistent with prior research. Previous studies also revealed that progesterone could suppress the expression of glucose transporter 4 (45), leading to increased insulin resistance (46), particularly in skeletal muscle as well as adipose tissue. In addition, progesterone may suppress different steps of the PI 3 kinase pathway, leading to increased insulin resistance and decreased glucose intake (47). Kin-Chuen Leung further validated the relationship and suggested that adiponectin and obesity may mediate the association between progesterone and insulin resistance (15). Significantly positive effects of progesterone on FPG and HbA1c were found in the current study, and these relationships were slightly altered but remained after gender stratification. Nevertheless, a positive association between progesterone and insulin resistance was not observed in the present study. The data of this study was derived from the baseline data of Henan Rural Cohort, which may result in a weak ability to detect such association. Participants taking glucoselowing medicine were ruled out in linear regression, which could lead to a weaker detected association than the actual relationship. Additionally, progesterone also accelerates the generation of other incretion (for example, noradrenaline) which elevate blood glucose (48). In terms of pregnenolone, it can be converted into progesterone in two steps in vivo, where the 3-hydroxyl group is oxidized to a keto group and then the double bond was transferred from position C-5 to C-4. This process is catalyzed by 3 beta hydroxysteroid dehydrogenase (3BHSD). Previous research noted that the inhibition of 3BHSD was accompanied by an improvement in glucose metabolism (including insulin resistance and C peptide) (49). As the activity of 3BHSD was inhibited, the conversion of pregnenolone to progesterone likely would be decreased, resulting in an accumulation of pregnenolone, which may explain the opposite direction of the impact of progesterone and pregnenolone on diabetes status.

The current study evaluated the opposing relationships of progesterone and pregnenolone with glucose homeostasis markers, modifiable prediabetes status, as well as T2DM; added new findings to these relationships; and provided evidence for the classification and intervention in individuals at high risk of diabetes in the Chinese population. Furthermore, this study derived data from a large ongoing cohort study based on a general population in rural region and collected information through a standardized protocol by trained interviewers, guaranteeing control over a wide range of potential confounders. Nevertheless, several limitations of this study should be noted. First, this case-control study was based on the baseline data of Henan Rural Cohort; therefore, the causal associations cannot be drawn in this study. Second, participants in this study were all from the Henan rural area, so the conclusions may not apply to urban populations. Third, although many confounding factors were considered and adjusted, the possibility of other potential confounders cannot be ruled out. Fourth, although opposite effect of progesterone and pregnenolone on diabetes status was observed, the mechanism is still unclear. Fifth, the evidence from preclinical study was limited. To the best of our knowledge, only one study evaluated the relationship of endogenous progesterone and diabetes in participants without pregnancy. However, similar results associated with progestogen-only contraceptives were consistent with the findings of this study. Sixth, an oral glucose tolerance test was not employed to diagnose prediabetes and T2DM. Finally, progestogen receptor function was not analyzed in this study, further limiting our ability to establish the mechanism of progestogen in diabetes.

\section{Conclusions}

In conclusion, the results of this study suggest that prediabetes and T2DM are positively linked to the serum concentration of progesterone, and negatively related to serum pregnenolone levels in Chinese rural men and postmenopausal women. In addition, interactive effects of progesterone and pregnenolone on prediabetes and T2DM were observed. Our findings indicate that progesterone and pregnenolone may play pivotal roles in the development of T2DM. Given the high prevalence and huge burden of diabetes, our findings may have an important implication for public health. However, these relationships remain to be validated in prospective multicenter studies, and the potential mechanism by which progestogen influence T2DM remains to be elucidated. Additionally, further studies are needed to explore the effect of progestogen receptor function on T2DM. 


\section{Supplementary data}

This is linked to the online version of the paper at https://doi.org/10.1530/ EJE-19-0352.

\section{Declaration of interest}

The authors declare that there is no conflict of interest that could be perceived as prejudicing the impartiality of this study.

\section{Funding}

This work was supported by the National Key Research and Development Program 'Precision Medicine Initiative' of China (grant numbers 2016YFC0900803); National Natural Science Foundation of China (grant numbers 21607136, 81573243, 81602925); and project was funded by China Postdoctoral Science Foundation (grant number 2016M602264). The funders had no role in the study design, data collection and analysis, decision to publish, or preparation of the manuscript.

\section{Ethics approval and consent to participate}

Ethics approval was obtained from the 'Zhengzhou University Life Science Ethics Committee', and written informed consent was obtained from all participants. Ethic approval code: (2015) MEC (S128).

\section{Acknowledgements}

Thanks to all participants in this study. Thanks for the research team who contributed to the data collection and laboratory measurement. Thanks for the valuable comments and advice from anonymous reviewers, and their suggestions help us to improve the current paper.

\section{References}

1 Zimmet P, Alberti KG \& Shaw J. Global and societal implications of the diabetes epidemic. Nature $2001 \mathbf{4 1 4}$ 782-787. (https://doi. org $/ 10.1038 / 414782 a)$

2 Stumvoll M, Goldstein BJ \& van Haeften TW. Type 2 diabetes: principles of pathogenesis and therapy. Lancet 2005365 1333-1346. (https://doi.org/10.1016/S0140-6736(05)61032-X)

3 GBD 2016 Disease and Injury Incidence and Prevalence Collaborators. Global, regional, and national incidence, prevalence, and years lived with disability for 328 diseases and injuries for 195 countries, 1990-2016: a systematic analysis for the Global Burden of Disease Study 2016. Lancet 2017390 1211-1259. (https://doi. org/10.1016/S0140-6736(17)32154-2)

$4 \mathrm{http}: / /$ diabetesatlas.org/key-messages.html. Accessed on 11 September 2018.

5 American Diabetes Association. Diagnosis and classification of diabetes mellitus. Diabetes Care 200932 (Supplement 1) S62-S67. (https://doi.org/10.2337/dc09-S062)

6 Wang L, Gao P, Zhang M, Huang Z, Zhang D, Deng Q, Li Y, Zhao Z, Qin X, Jin D et al. Prevalence and ethnic pattern of diabetes and prediabetes in China in 2013. JAMA 2017317 2515-2523. (https:// doi.org/10.1001/jama.2017.7596)

7 Liu X, Li Y, Li L, Zhang L, Ren Y, Zhou H, Cui L, Mao Z, Hu D \& Wang C. Prevalence, awareness, treatment, control of type 2 diabetes mellitus and risk factors in Chinese rural population: the RuralDiab study. Scientific Reports 20166 31426. (https://doi.org/10.1038/ srep31426)

8 Joseph JJ, Echouffo-Tcheugui JB, Kalyani RR, Yeh HC, Bertoni AG, Effoe VS, Casanova R, Sims M, Correa A, Wu WC et al. Aldosterone, renin, and diabetes mellitus in African Americans: the Jackson heart study. Journal of Clinical Endocrinology and Metabolism 2016101 1770-1778. (https://doi.org/10.1210/jc.2016-1002)

9 Hackett RA, Kivimaki M, Kumari M \& Steptoe A. Diurnal cortisol patterns, future diabetes, and impaired glucose metabolism in the Whitehall II cohort study. Journal of Clinical Endocrinology and Metabolism 2016101 619-625. (https://doi.org/10.1210/jc.2015-2853)

10 Mayes JS \& Watson GH. Direct effects of sex steroid hormones on adipose tissues and obesity. Obesity Reviews 20045 197-216. (https:// doi.org/10.1111/j.1467-789X.2004.00152.x)

11 Campbell SE \& Febbraio MA. Effects of ovarian hormones on exercise metabolism. Current Opinion in Clinical Nutrition and Metabolic Care 20014 515-520. (https://doi.org/10.1097/00075197-20011100000009)

12 Sharkey LC, Kirchain S, McCune SA, Simpson GI, Archambault EZ, Boatright NK, Hicks E \& Fray J. Progesterone increases blood pressure in spontaneous gestational hypertension in rats. American Journal of Hypertension 200518 36-43. (https://doi.org/10.1016/j. amjhyper.2004.07.024)

13 Nunes VA, Portioli-Sanches EP, Rosim MP, Araujo MS, PraxedesGarcia P, Valle MM, Roma LP, Hahn C, Gurgul-Convey E, Lenzen S et al. Progesterone induces apoptosis of insulin-secreting cells: insights into the molecular mechanism. Journal of Endocrinology 2014 221 273-284. (https://doi.org/10.1530/JOE-13-0202)

14 Branisteanu DD \& Mathieu C. Progesterone in gestational diabetes mellitus: guilty or not guilty? Trends in Endocrinology and Metabolism 200314 54-56. (https://doi.org/10.1016/S1043-2760(03)00003-1)

15 Leung KC, Xu A, Craig ME, Martin A, Lam KS \& O'Sullivan AJ. Adiponectin isoform distribution in women - relationship to female sex steroids and insulin sensitivity. Metabolism: Clinical and Experimental 200958 239-245. (https://doi.org/10.1016/j. metabol.2008.09.020)

16 Picard F, Wanatabe M, Schoonjans K, Lydon J, O’Malley BW \& Auwerx J. Progesterone receptor knockout mice have an improved glucose homeostasis secondary to beta-cell proliferation. PNAS 2002 99 15644-15648. (https://doi.org/10.1073/pnas.202612199)

17 Morley P, Calaresu FR, Barbe GJ \& Armstrong DT. Insulin enhances luteinizing hormone-stimulated steroidogenesis by porcine theca cells. Biology of Reproduction 198940 735-743. (https://doi. org/10.1095/biolreprod40.4.735)

18 Xiang AH, Kawakubo M, Kjos SL \& Buchanan TA. Long-acting injectable progestin contraception and risk of type 2 diabetes in Latino women with prior gestational diabetes mellitus. Diabetes Care 200629 613-617. (https://doi.org/10.2337/diacare.29.03.06.dc051940)

19 Xiang AH, Kjos SL, Takayanagi M, Trigo E \& Buchanan TA. Detailed physiological characterization of the development of type 2 diabetes in Hispanic women with prior gestational diabetes mellitus. Diabetes 201059 2625-2630. (https://doi.org/10.2337/db10-0521)

20 Glisic M, Shahzad S, Tsoli S, Chadni M, Asllanaj E, Rojas LZ, Brown E, Chowdhury R, Muka T \& Franco OH. Association between progestinonly contraceptive use and cardiometabolic outcomes: a systematic review and meta-analysis. European Journal of Preventive Cardiology 201825 1042-1052. (https://doi.org/10.1177/2047487318774847)

21 Pergialiotis V, Bellos I, Hatziagelaki E, Antsaklis A, Loutradis D \& Daskalakis G. Progestogens for the prevention of preterm birth and risk of developing gestational diabetes mellitus: a meta-analysis. American Journal of Obstetrics and Gynecology 2019. (https://doi. org/10.1016/j.ajog.2019.05.033)

22 Nilsson SE, Fransson E \& Brismar K. Relationship between serum progesterone concentrations and cardiovascular disease, diabetes, and mortality in elderly Swedish men and women: an 8-year prospective study. Gender Medicine 20096 433-443. (https://doi. org/10.1016/j.genm.2009.09.011)

23 Liu X, Mao Z, Li Y, Wu W, Zhang X, Huo W, Yu S, Shen L, Li L, Tu R et al. The Henan Rural Cohort: a prospective study of chronic non- 
communicable diseases. International Journal of Epidemiology 2019 Epub. (https://doi.org/10.1093/ije/dyz039)

24 World Health Organization. Definition, Diagnosis and Classification of Diabetes Mellitus and Its Complications. Part 1: Diagnosis and Classification of Diabetes Mellitus, 2nd ed. Geneva: World Health Organization, 1999.

25 Fan M, Lyu J \& He P. Chinese guidelines for data processing and analysis concerning the International Physical Activity Questionnaire. Zhonghua Liu Xing Bing Xue Za Zhi 201435 961-964.

26 Wallace TM, Levy JC \& Matthews DR. Use and abuse of HOMA modeling. Diabetes Care 200427 1487-1495. (https://doi. org/10.2337/diacare.27.6.1487)

27 Sun X, Li J, Jin S, Li Y, Liu W, Zhao H, Zhou Y, Jiang Y, Liu H, $\mathrm{Xia} \mathrm{W}$ et al. Associations between repeated measures of maternal urinary phthalate metabolites during pregnancy and cord blood glucocorticoids. Environment International 2018121 471-479. (https://doi.org/10.1016/j.envint.2018.09.037)

28 Liu X, Jiang J, Liu X, Luo Z, Wang Y, Dong X, Wei D, Li R, Wang Y, Huo $\mathrm{W}$ et al. Association of serum testosterone with different classes of glucose metabolism and the mediation effect of obesity: the Henan Rural Cohort Study. Diabetes/Metabolism Research and Reviews 201935 e3133. (https://doi.org/10.1002/dmrr.3133)

29 Lamina C, Sturm G, Kollerits B \& Kronenberg F. Visualizing interaction effects: a proposal for presentation and interpretation. Journal of Clinical Epidemiology 201265 855-862. (https://doi. org/10.1016/j.jclinepi.2012.02.013)

30 Kjos SL, Peters RK, Xiang A, Thomas D, Schaefer U \& Buchanan TA. Contraception and the risk of type 2 diabetes mellitus in Latina women with prior gestational diabetes mellitus. JAMA $1998 \mathbf{2 8 0}$ 533-538. (https://doi.org/10.1001/jama.280.6.533)

31 Kim C, Seidel KW, Begier EA \& Kwok YS. Diabetes and depot medroxyprogesterone contraception in Navajo women. Archives of Internal Medicine 2001161 1766-1771. (https://doi.org/10.1001/ archinte.161.14.1766)

32 Kahn HS, Curtis KM \& Marchbanks PA. Effects of injectable or implantable progestin-only contraceptives on insulin-glucose metabolism and diabetes risk. Diabetes Care 200326 216-225. (https://doi.org/10.2337/diacare.26.1.216)

33 Bender NM, Segall-Gutierrez P, Najera SO, Stanczyk FZ, Montoro M \& Mishell Jr DR. Effects of progestin-only long-acting contraception on metabolic markers in obese women. Contraception 201388 418-425. (https://doi.org/10.1016/j.contraception.2012.12.007)

34 Lopez LM, Grimes DA \& Schulz KF. Steroidal contraceptives: effect on carbohydrate metabolism in women without diabetes mellitus. Cochrane Database of Systematic Reviews 20094 CD006133. (https:// doi.org/10.1002/14651858.CD006133.pub5)

35 Rebarber A, Istwan NB, Russo-Stieglitz K, Cleary-Goldman J, Rhea DJ, Stanziano GJ \& Saltzman DH. Increased incidence of gestational diabetes in women receiving prophylactic 17alphahydroxyprogesterone caproate for prevention of recurrent preterm delivery. Diabetes Care 200730 2277-2280. (https://doi.org/10.2337/ dc07-0564)

36 Romero R \& Stanczyk FZ. Progesterone is not the same as 17alphahydroxyprogesterone caproate: implications for obstetrical practice. American Journal of Obstetrics and Gynecology 2013208 421-426. (https://doi.org/10.1016/j.ajog.2013.04.027)
37 Waters TP, Schultz BA, Mercer BM \& Catalano PM. Effect of 17alphahydroxyprogesterone caproate on glucose intolerance in pregnancy. Obstetrics and Gynecology 2009114 45-49. (https://doi.org/10.1097/ AOG.0b013e3181a9454b)

38 Figueroa-Valverde L, Diaz-Cedillo F, Lopez-Ramos M, GarciaCervera E, Pool-Gomez E, Cardena-Arredondo C \& Ancona-Leon G. Glibenclamide-pregnenolone derivative has greater hypoglycemic effects and biodistribution than glibenclamide- $\mathrm{OH}$ in alloxan-rats. Biomedical Papers of the Medical Faculty of the University Palacky, Olomouc, Czech Republic 2012156 122-127. (https://doi.org/10.5507/ bp.2012.028)

39 Vermeulen A. The hormonal activity of the postmenopausal ovary. Journal of Clinical Endocrinology and Metabolism 197642 247-253. (https://doi.org/10.1210/jcem-42-2-247)

40 Blanchette S, Marceau P, Biron S, Brochu G \& Tchernof A. Circulating progesterone and obesity in men. Hormone and Metabolic Research 200638 330-335. (https://doi.org/10.1055/s-2006-925392)

41 Hofmann A, Peitzsch M, Brunssen C, Mittag J, Jannasch A, Frenzel A, Brown N, Weldon SM, Eisenhofer G, Bornstein SR et al. Elevated steroid hormone production in the $\mathrm{db} / \mathrm{db}$ mouse model of obesity and type 2 diabetes. Hormone and Metabolic Research 201749 43-49. (https://doi.org/10.1055/s-0042-116157)

$42 \mathrm{Li}$ Y, Zhao L, Yu D \& Ding G. The prevalence and risk factors of dyslipidemia in different diabetic progression stages among middleaged and elderly populations in China. PLoS ONE 201813 e0205709. (https://doi.org/10.1371/journal.pone.0205709)

43 Lenzen S \& Bailey CJ. Thyroid hormones, gonadal and adrenocortical steroids and the function of the islets of Langerhans. Endocrine Reviews 19845 411-434. (https://doi.org/10.1210/edrv-5-3-411)

44 Straub SG, Sharp GW, Meglasson MD \& De Souza CJ. Progesterone inhibits insulin secretion by a membrane delimited, nongenomic action. Bioscience Reports 200121 653-666. (https://doi. org/10.1023/a:1014773010350)

45 Sugaya A, Sugiyama T, Yanase S, Shen XX, Minoura H \& Toyoda N. Expression of glucose transporter 4 mRNA in adipose tissue and skeletal muscle of ovariectomized rats treated with sex steroid hormones. Life Sciences 200066 641-648. (https://doi.org/10.1016/ s0024-3205(99)00636-0)

46 Butte NF. Carbohydrate and lipid metabolism in pregnancy: normal compared with gestational diabetes mellitus. American Journal of Clinical Nutrition 200071 (5 Supplement) 1256S-1261S. (https://doi. org/10.1093/ajcn/71.5.1256s)

47 Wada T, Hori S, Sugiyama M, Fujisawa E, Nakano T, Tsuneki H, Nagira K, Saito S \& Sasaoka T. Progesterone inhibits glucose uptake by affecting diverse steps of insulin signaling in 3T3-L1 adipocytes. American Journal of Physiology. Endocrinology and Metabolism 2010298 E881-E888. (https://doi.org/10.1152/ajpendo.00649.2009)

48 Wilsterman K, Pepper A \& Bentley GE. Low glucose availability stimulates progesterone production by mouse ovaries in vitro. Journal of Experimental Biology 2017220 4583-4588. (https://doi. org/10.1242/jeb.164384)

49 Vrbikova J, Hill M, Starka L, Cibula D, Bendlova B, Vondra K, Sulcová J \& Snajderová M. The effects of long-term metformin treatment on adrenal and ovarian steroidogenesis in women with polycystic ovary syndrome. European Journal of Endocrinology 2001 144 619-628. (https://doi.org/10.1530/eje.0.1440619)

Received 7 May 2019

Revised version received 9 September 2019

Accepted 2 October 2019 